\title{
GLOSSary: the GLobal Ocean $16 S$ subunit web accessible resource
}

\author{
M. Tangherlini ${ }^{1 *}$, M. Miralto $^{1 \dagger}$, C. Colantuono ${ }^{1}$, M. Sangiovanni', A. Dell' Anno ${ }^{2}$, C. Corinaldesi ${ }^{3}$, R. Danovaro ${ }^{1,2}$ \\ and M. L. Chiusano ${ }^{1,4}$
}

From BBCC Conference 2017

Naples, Italy. 18 - 20 December 2017

\begin{abstract}
Background: Environmental metagenomics is a challenging approach that is exponentially spreading in the scientific community to investigate taxonomic diversity and possible functions of the biological components. The massive amount of sequence data produced, often endowed with rich environmental metadata, needs suitable computational tools to fully explore the embedded information. Bioinformatics plays a key role in providing methodologies to manage, process and mine molecular data, integrated with environmental metagenomics collections. One such relevant example is represented by the Tara Ocean Project.

Results: We considered the Tara $16 \mathrm{~S}$ miTAGs released by the consortium, representing raw sequences from a shotgun metagenomics approach with similarities to $16 \mathrm{~S}$ rRNA genes. We generated assembled $16 \mathrm{~S}$ rDNA sequences, which were classified according to their lengths, the possible presence of chimeric reads, the putative taxonomic affiliation. The dataset was included in GLOSSary (the GLobal Ocean 165 Subunit web accessible resource), a bioinformatics platform to organize environmental metagenomics data. The aims of this work were: i) to present alternative computational approaches to manage challenging metagenomics data; ii) to set up user friendly web-based platforms to allow the integration of environmental metagenomics sequences and of the associated metadata; iii) to implement an appropriate bioinformatics platform supporting the analysis of $16 \mathrm{~S}$ rDNA sequences exploiting reference datasets, such as the SILVA database. We organized the data in a next-generation NOSQL "schema-less" database, allowing flexible organization of large amounts of data and supporting native geospatial queries. A web interface was developed to permit an interactive exploration and a visual geographical localization of the data, either raw miTAG reads or $16 \mathrm{~S}$ contigs, from our processing pipeline. Information on unassembled sequences is also available. The taxonomic affiliations of contigs and miTAGs, and the spatial distribution of the sampling sites and their associated sequence libraries, as they are contained in the Tara metadata, can be explored by a query interface, which allows both textual and visual investigations. In addition, all the sequence data were made available for a dedicated BLAST-based web application alongside the SILVA collection.
\end{abstract}

Conclusions: GLOSSary provides an expandable bioinformatics environment, able to support the scientific community in current and forthcoming environmental metagenomics analyses.

Keywords: Tara Ocean project, Marine metagenomics, 165 rRNA, Taxonomic analysis

\footnotetext{
*Correspondence: michael.tangherlini@szn.it

${ }^{+} \mathrm{M}$. Tangherlini and M. Miralto contributed equally to this work.

'Stazione Zoologica "Anton Dohrn", Villa Comunale, 80121 Naples, Italy

Full list of author information is available at the end of the article
}

(c) The Author(s). 2018 Open Access This article is distributed under the terms of the Creative Commons Attribution 4.0 International License (http://creativecommons.org/licenses/by/4.0/), which permits unrestricted use, distribution, and reproduction in any medium, provided you give appropriate credit to the original author(s) and the source, provide a link to the Creative Commons license, and indicate if changes were made. The Creative Commons Public Domain Dedication waiver (http://creativecommons.org/publicdomain/zero/1.0/) applies to the data made available in this article, unless otherwise stated. 


\section{Background}

Environmental metagenomics is a challenging approach that has rapidly expanded in the last decade thanks to advanced high throughput sequencing technologies. Massive amounts of sequence data are being produced, accompanied by information annotating spatial localization of the sampling sites, as well as other useful data related to the environmental conditions (e.g. nutrient concentrations, temperature [1]), which represent some of the driving factors that can shape community structures by resource partitioning [2] and influence biological processes [3].

Metagenomic approaches are mainly focused on the analysis of microbial communities, fostering a deeper characterization of prokaryotic and eukaryotic diversity. The exploration of marine microbial communities is expanding to integrate classical phylogenetic analyses with information from environmental data and spatial-temporal variation of such communities [4]. Important efforts, indeed, aims at considering multilevel aspects, such as molecular, biological, physical, and chemical data for holistic approaches to the study and the understanding of the processes which regulate Earth's biogeochemical cycles and climate [5]. The global ocean exploration that started in the last two decades is contributing enormously to this aim. The Sorcerer II expeditions (2003-2010) and the Malaspina expedition (2010-2011) carried out surveys for exploring microbial diversity from the ocean surface down to bathyal depths $(>1,000 \mathrm{~m})$. The Tara Oceans Expedition (2009-2013) is by far the most recent and largest expedition, aimed at investigating microbial and eukaryotic diversity at global scale [5]. These research efforts result in the production of a huge amount of data; as an example, the Tara Oceans expedition resulted in the collection of over 35,000 samples of seawater and plankton, with most of the data represented by sequence data [6]. The analysis of such "Big Data" collections requires huge collective efforts, addressed by several and independent research teams [6-11]. However, the amount of produced sequence data requires dedicated infrastructures to be fully exploited and properly analysed, and this represents one of the most daunting challenges in the field of environmental ecology. Organising such collections and their precious and multifaceted information content by suitable bioinformatic tools represents a key step to foster both data mining and collaborative research and discovery $[5,6]$, with relevant impact for a better understanding of microbial diversity and factors influencing its distribution.

Previous attempts at the creation of sequence storage and analytical platforms, dedicated to the study of environmental sequence data, included the CAMERA database [12], which is now discontinued; the VAMPS system [13], for ribosomal sequence analysis; the MG-RAST server for metagenomics and metatranscriptomics [14]; and QIITA [15], which acts as a sequence analysis platform serving as a repository for the Earth Microbiome Project [16]. However, none of them currently supports metadata-enabled dataset exploration.

To test a novel computational framework for the management and the analysis of environmental omics collections, we designed a document-oriented schema-less database together with a web interface with the aim of setting up: i) a suite using alternative computational approaches to manage challenging metagenomics data; ii) a user-friendly web-based platform to allow the integration of environmental metagenomics sequences and of the associated metadata; iii) an appropriate bioinformatics platform to support the analysis of $16 \mathrm{~S}$ rDNA sequences while exploiting reference datasets, such as the SILVA data collection [17].

Although public sequence collections have been recently distributed through the European Nucleotide Archive [18] by the Tara Ocean consortium, 16S sequence data are only available in the form of raw reads, also providing a basic summary of the associated diversity. However, to our knowledge, no sequence curation is provided yet. Therefore, we decided to start the implementation of the platform here proposed including data from this relevant collection. We considered the $16 \mathrm{~S}$ miTAGs [8, 19] originally retrieved from shotgun sequencing data by means of specialized HMM-based pipelines focused on ribosomal $16 \mathrm{~S}$ rRNA gene sequences within the $0.22-1.6 \mu \mathrm{m} / 0.22-3 \mu \mathrm{m}$ size fractions of water samples processed by the Tara Ocean consortium. We processed the miTAGs data, and classified complete or partial sequences, as well as potential chimeric and chimera-free sequences, providing taxonomic affiliation by means of the SILVA 16 S gene sequence database. We organized all the sequence data and their associated metadata in the platform and designed suitable analytical tools to browse the entire collection, aiming to support in-depth exploitation of this precious resource.

\section{Implementation}

Data availability

Tara miTAGs libraries, representing $16 \mathrm{~S}$ rDNA sequence tags extracted from Tara raw reads (thus not including $18 \mathrm{~S}$ sequence data), as well as all the metadata related to all libraries and to the specific Tara stations, were downloaded from the Tara Ocean companion website (http://ocean-microbiome.embl.de/ companion.html). MiTAG libraries were produced from shotgun sequence data from the $0.22-1.6 \mu \mathrm{m} /$ $0.22-3 \mu \mathrm{m}$ size fractions of water samples generated 
by the Tara Ocean consortium, by means of specialized HMM-based pipelines focused on prokaryotic ribosomal 16S genes. Sampling depths are reported per library and correspond to surface oceanic waters ("SRF"), epipelagic waters ("MIX"), waters sampled at the deep-chlorophyll maximum ("DCM") and mesopelagic waters ("MES") [6, 7]; libraries were assigned to stations, depth layers, marine biomes and oceanic provinces according to the metadata provided in [6].

\section{Assembly and annotation pipeline}

MiTAG sequences from each library were independently assembled by the MEGAHIT software [20]. MEGAHIT was chosen because it achieves a reasonable resolution of 16S rRNA genes micro-diversity (intended as $16 \mathrm{~S}$ gene sequence variants with an overall similarity higher than 97\%, e.g. 1-3 nucleotides) during the assembly, as reported in [21]. MEGAHIT was used with the following settings: k-mer lengths from 21 to 99 (with a k-step of 10) and a minimum coverage of 2 sequences (to maximize assembly sensitivity). Other parameters were left as default. This allowed us to discern prokaryotic strains potentially differing for few nucleotides in their $16 \mathrm{~S}$ genes, thus broadening our view of prokaryotic diversity in marine habitats.

To detect sequences presumably used to build each assembly, and remove sequences with ambiguous mapping, miTAG sequences from each library were indipendently mapped to all assembled contigs using the BBMap tool (https://sourceforge.net/projects/bbmap/), with a minimum identity threshold of $97 \%(\operatorname{minid}=97)$ and all the other in the default settings. Mapped and unmapped (i.e. singletons) sequences were therefore associated to each library from each Tara station and depth. The percentage of mapped miTAGs, i.e. the number of miTAGs mapped to each contig versus the total library size, was used as a proxy for defining the relative abundance of a contig in a library. This also represents the coverage of the contig, and is reported in the header associated to each contig sequence when exploring the database. Visualization of the resulting data and statistical analyses were performed within the $\mathrm{R}$ environment [22].

To trim the assembled contigs and remove non-16S portions, the $s s u \_f i n d e r$ function of the CheckM package [23] was used. From this dataset, all sequences with a minimum length of $900 \mathrm{bp}$ were kept using the PRINSEQ tool [24] and were defined as "long" sequences; sequences with a length ranging from 800 to $899 \mathrm{bp}$ were also recovered by the same tool and classified as "medium". Sequences shorter than 800 nucleotides, that confirmed their similarity to $16 \mathrm{~S}$ RNAs, were defined as "short."
Long and medium contigs were annotated using the assign_taxonomy function of the QIIME package (v1.9; [25]) using the SILVA v128 [17] database as a reference. Both sets of contigs were independently checked for possible chimeras using the VSEARCH package [26], again using the SILVA v128 database as a reference. We therefore classified all long and medium contigs as chimeric ("chimera"), non-chimeric ("chimera free") and "borderline", accordingly.

Taxonomy affiliations for short contigs $(<800 \mathrm{bp})$, as well as for the miTAG sequences, were obtained by a similarity search versus the SILVA database using the VSEARCH package using an identity cutoff of $80 \%$ and keeping the best hits. The complete pipeline is shown in Fig. 1.

\section{Database organization and query system}

The database was implemented using MongoDB [27], a document-oriented NoSQL Database management system. Indeed, MongoDB permits to efficiently store and retrieve semantically similar data having highly different structures. More in detail, the database underlying GLOSSary holds together miTAG and contig sequences (i.e. semantically similar objects), although each type could have a largely different metadata set (i.e. structure). Data were de-normalized and split over several collections, taking into account query performances as one of the main criteria. Starting from a pre-defined set of queries and data-browsing requirements, data collections were built together with a first set of indexes to allow fast data retrieval. The sequences were stored in a dedicated collection. Each sequence data object was augmented with a set of metadata, which differ across miTAG or contig sequences. The sequences were then tagged using an indexed array of keywords, representing query filtering criteria exploited in the search engine. It is worth noting that each sequence object can be used to store large amount of data (up to $16 \mathrm{MB}$, as for the default per-document object limit in MongoDB). Due to the unstructured nature of the available data, a mix of single field indexes, geospatial indexes and text indexes were defined for each data collection to optimize data querying. To speed-up searches on large set of sequences (in the order of Millions of objects) a query caching approach was developed. The database general schema is represented in Fig. 2. The database can be accessed by a suitable query system through a web interface realized with a Pyramid stack (https://trypyramid.com/), a Python-based web framework. The web frontend was developed using HTML5, Bootstrap (https://getbootstrap.com/) and Jquery (https://jquery.com/).

\section{BLAST service}

A BLAST service was implemented to allow similarity searches on raw $16 \mathrm{~S}$ miTAG sequences, the contigs and 


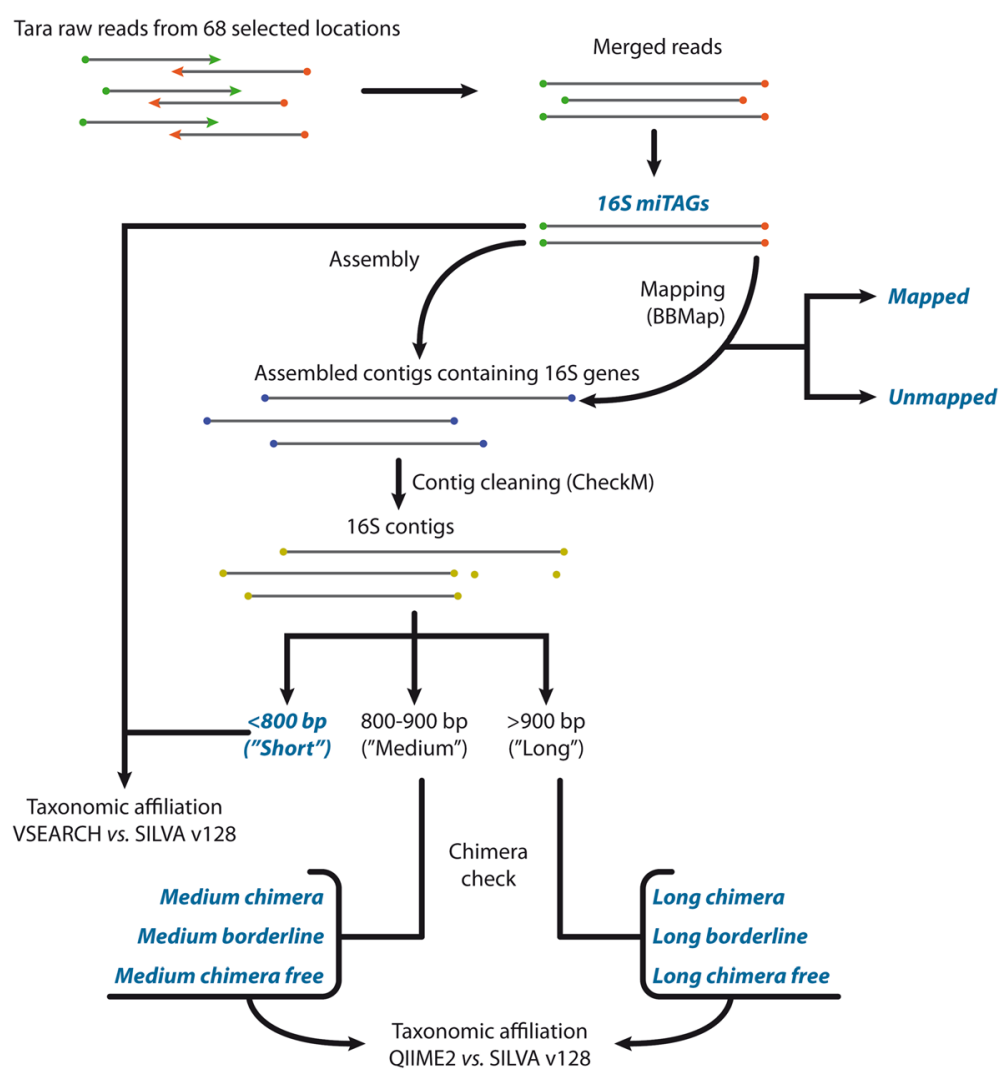

Fig. 1 Schematic representation of the pipeline implemented to process and annotate the data included in the GLOSSary database. Data included in the database and their classification are indicated in blue

the 16S full-length gene sequences from the SILVA database (v128) [17]. The service was developed using the Sequence Server software [28].The BLAST service allows the user to compare one or more query sequences (provided in FASTA format) against different partitions of the data collections included in the platform. The resulting hits are cross-linked to the web platform above described.

\section{Results}

\section{Data content}

The total amount of sequencing data associated with the 139 miTAG libraries available on the Tara Ocean portal corresponded to $14,129,971$ miTAG sequences. Of these, $3,489,675$ reads (representing, on average, ca. $25 \%$ of the raw miTAGs in each library) were assembled in contigs, according to the in-house implemented pipeline (Fig. 1). On average, ca. $5 \%$ of the total resulting contigs $(103,954$ sequences) was greater or equal to $800 \mathrm{bp}$ in length and identified as potentially non-chimeric. An additional table shows this in more detail (Additional file 1).

The number of miTAG libraries obtained for each marine province of the Tara sampling was very variable, with the highest number of libraries (27) in the South
Pacific Subtropical Gyre Province (SPSG). Surface layers ("SRF") were represented across all provinces, but deeper layers (i.e. deep-chlorophyll maximum ("DCM"), epipelagic mixed waters ("MIX") and mesopelagic waters ("MES")) were not: indeed, a total of 63 SRF, 42 DCM and 30 MES libraries were produced, and some layers were entirely missing (e.g. the DCM layer from the Gulf Stream province) A visualization of this unevenness is shown in an additional figure (Additional file 2).

The number of sequences included in each miTAG library was highly variable (Additional file 1), ranging from the small content of 39410 sequences in the TARA-066-DCM library (Benguela province, DCM layer) to the 186,898 sequences in the TARA-064-SRF library (Eastern Africa Coastal province, SRF layer). However, more than $24 \%$ of the sequences across all libraries (up to $43 \%$ ) were successfully assembled.

\section{Platform organization and accessibility}

The platform is organized in two independent partitions: 1) a database including sequence data and metadata, and 2) a BLAST based platform including miTAGs and contigs, together with the $16 \mathrm{~S}$ full-length sequences from the SILVA database (v128). 


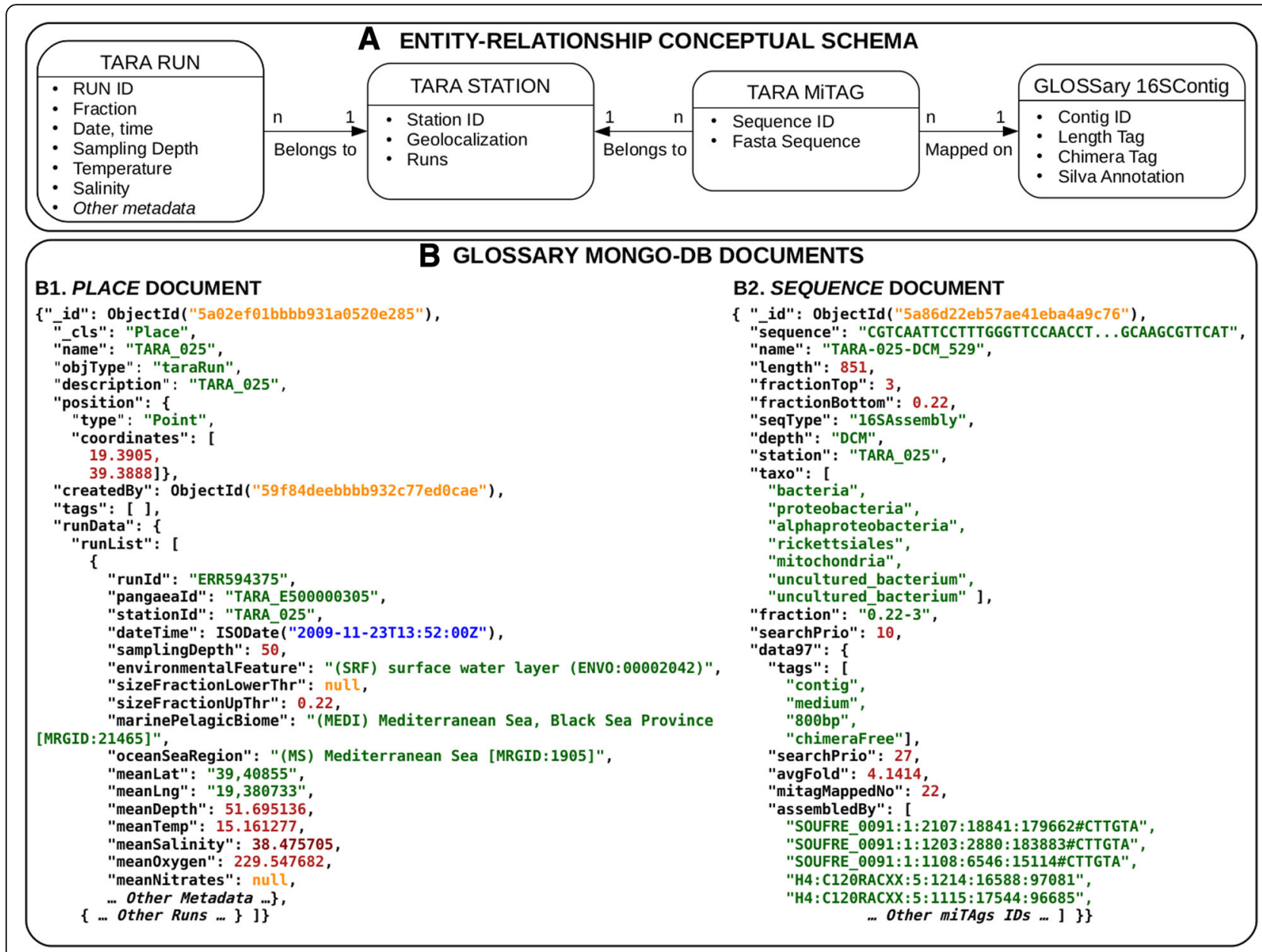

Fig. 2 Conceptual and implemented architecture of the GLOSSary database. a Entity-Relationship diagram of the GLOSSary data. The Tara sequences and metadata are organised in runs (TARA RUM), each belonging to a specific station (TARA STATIOM). TARA MiTAGs are short sequences obtained by merging the paired end reads matching on the Silva 165 database, grouped by station, depth and fraction. The GLOSSary 16SContigs are longer sequences obtained by re-assemblying the MiTAGs with the glossary pipeline, and mapping them to the Silva database. $\mathbf{b}$ The GLOSSary Mongo-db document organization. The data presented in panel A have been denormalised and reorganised in two main documents: PLACE (B1) and SEQUENCE (B2). The PLACE document holds all the information on the Tara stations and associated runs and metadata. The SEQUENCE document holds the GLOSSary 165 contigs and the unassamblied MiTAGS nucleotidic sequences, together with the associated metadata and, for the 16SContigs, the taxonomical information and assembled MiTAGS identifiers

All raw and assembled sequences (i.e. miTAGs and contigs) were uploaded into a MongoDB Database management system (DBMS). The sequences are characterized by a "tag", i.e. a descriptive string reporting the classification of several features such as: i) mapped and unmapped status for each miTAG; ii) contig classification (long, medium and short) and iii) contig contamination level (chimera free, chimera and borderline). Information about the metadata (such as Tara station ID, depth and fraction) associated to each miTAG were kept and used as added "tags".

Both the miTAGs and the $16 \mathrm{~S}$ contigs can be explored through the web page http://bioinfo.szn.it/glossary (Fig. 3a). Two main sections are accessible: a "Query Search" page (Fig. 3b) and a "BLAST Search" service (Fig. 3c).
The "BLAST Search" service allows the user to compare one or more query sequences (to be provided in FASTA format) against different subsets of the data included in the GLOSSARY database. Moreover, among the datasets made available in the BLAST partition, we also included the SILVA $16 \mathrm{~S}$ full-length sequences, to allow cross comparisons between the entire data collection organized in the database and a reference database (Fig. 3c). The user can select one or more of the included collections, and the alignments in the classical BLAST output format are shown.

In the "Query Search" area of the platform, three main entry points are currently available. The "Search by taxonomy" allows a query by keyword based on taxonomical classification; the "Search by sequence ID" allows to use 


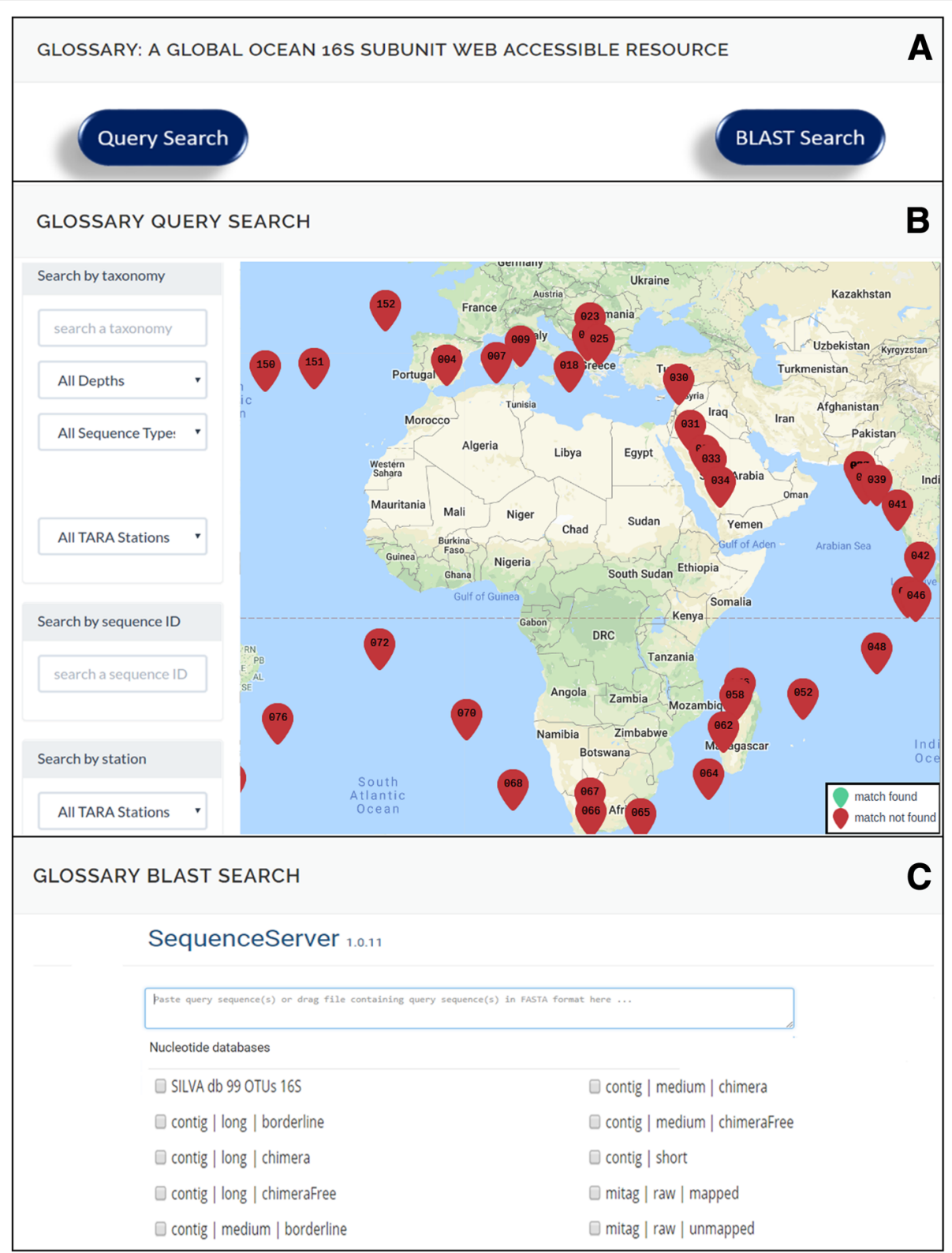

Fig. 3 The GLOSSary web interface. a Links to the "Query Search" and the "BLAST Search" are accessible by the dark blue boxes in the main page of the GLOSSary platform. b The "Query Search" main page: "Search by taxonomy", "Search by sequence ID" and "Search by station" are shown. Red pointers on the interactive geographic map indicate the Tara stations by number. Each pointer is linked to the station information details. c The "BLAST search" main page. The sequence server interface permits to perform BLAST searches on one or multiple data subsets (Tags) of the GLOSSary database. The SILVA database is also querable in this partition

miTAGs or contigs identifiers to access each single sequence features; the "Search by station" allows to access to all the runs and the miTAGs belonging each Tara station.

An interactive geographic map can be also exploited independently, or can accompany each query by the appropriate result. The map changes according to the results of each query, highlighting in green all stations containing sequences associated with the searched keyword, thus permitting an immediate and dynamic view of the geographical distribution of the stations associated to each query.

In detail, the "Search by taxonomy" query field allows to perform the search specifying the available taxonomic affiliations while typing one or more chars. A dropdown list appears accordingly supporting the selection. The list includes all the taxa that are associated to at least one of the sequences included in the database (Fig. 4a). Furthermore, it is possible to further specify the query by selecting: i) the sampling 
depth: "Surface Oceanic" ("SRF"), "Deep-Chlorophyll Maximum" ("DCM"), "Epipelagic" ("MIX") and "Mesopelagic" ("MES"); ii) the sequence type, i.e. "All Sequence Types", "miTAGs" or "16S Contigs"; iii) when searching for "16S Contigs" it is possible furthermore to select the contigs by length ("All Contigs Length", "Long (> 900bp)", "Medium (> 800bp and < 900bp)", "Short (< $800 \mathrm{bp})$ "); iv) when searching for "miTAGs" it is possible to choose among "All miTAGs", "Mapped" or "Unmapped" sequences, thus identifying all sequences types including those that resulted as singletons at the given similarity threshold; v) the
Tara station ID: "All TARA Stations" or, selecting from the dropdown list, a specific station ID.

The query results are visualized next to the search boxes and provide information on the total number of hits and stations found for the selected criteria (topmost line, Fig. 4a) and a table, showing the number of hits found for each tag. Result sequences are downloadable in a FASTA formatted file (Fig. 4a), whose headers contain information on the Tara station from which the sequence was reconstructed, alongside with size fraction, depth layer, taxonomy, sequence tags and coverage information. By clicking with the mouse on the "total

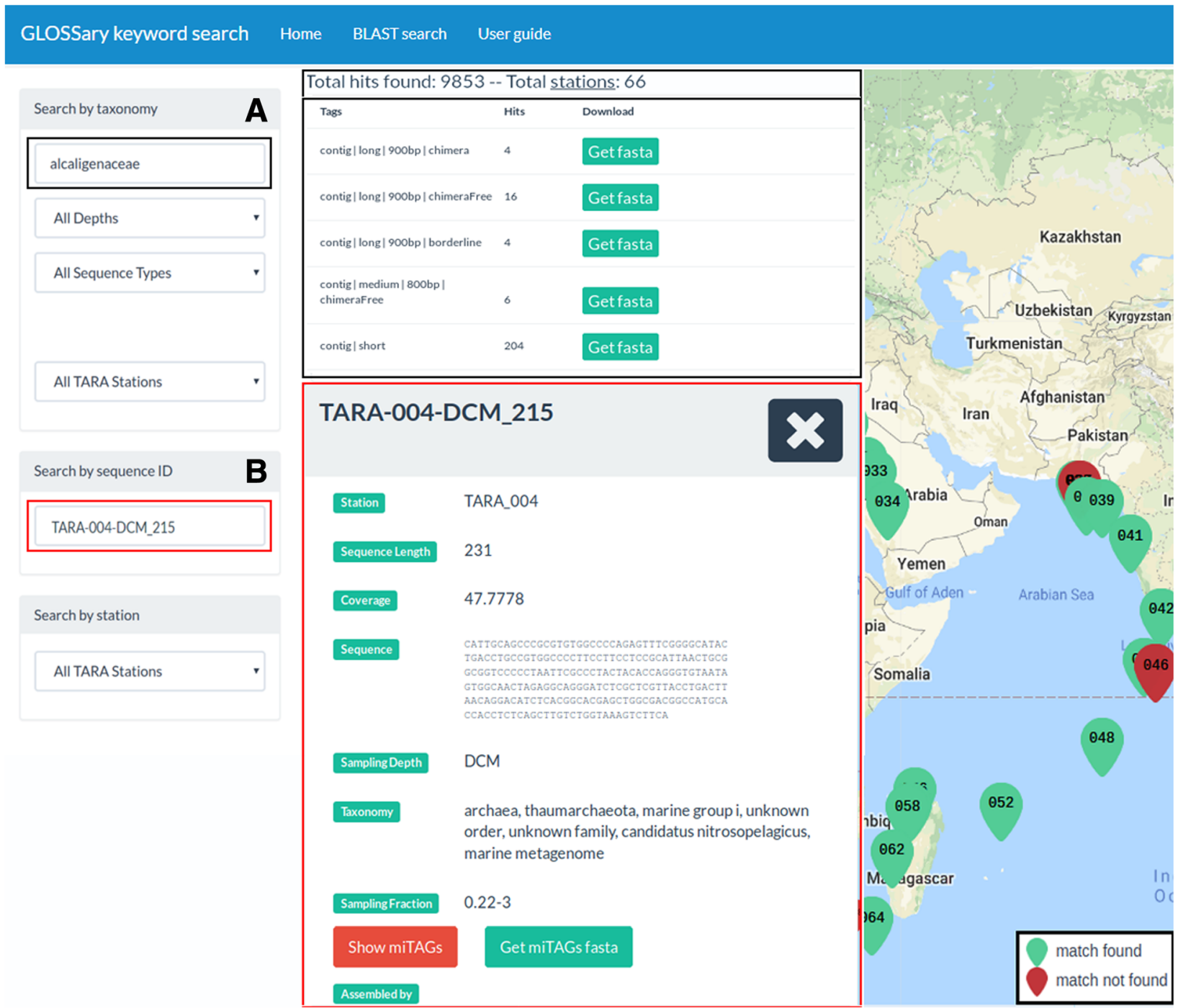

Fig. 4 Details on example queries. a Selecting in the "Search by taxonomy" section the "Alcaligenaceae", "All Depths", "All Sequence Types" and "All TARA Stations" options, the total number of elements found for each Tag is shown. A button allows to download the sequences belonging to each Tag ("Get fasta" green boxes). The Tara stations associated to each query are also shown on the map by green pointers. b Typing in the "Search by sequence ID" field the "TARA-004-DCM_215" contig ID, several details are shown including the sequence, its length, the taxonomic affiliation, the Tara station it belongs to, the sampling depth, the sampling fraction. It is also possible to view the list of all the miTAGs assembled on that contig ("Show miTAGs" red box), or to download the corresponding miTAGs FASTA file ("Get miTAGs fasta" green box) 
stations" link in the summary line, it is possible to visualize the list of hit counts for each station.

By searching for a specific sequence ID in the "Search by sequence ID" box it is possible to obtain information on the sequence and its length, the corresponding Tara station, the sampling depth and fraction, and the complete taxonomic affiliation. In the case of contigs the coverage is available, and the miTAGs sequences mapped at the specific threshold are also accessible, whereas, in the case of miTAGs, their status in terms of mapped or unmapped to contigs is reported too (Fig. 4b).

Finally, searching by Tara station IDs in the dropdown menu of the "Search by station" box allows to obtain the list of the raw runs for each station, each linked to the NCBI SRA download page, as well as the list of the miTAGs sequences (as FASTA archives) organised by depth (Fig. 5).

For all the search types, the resulting stations are highlighted in green on the map. The map itself can be interactively explored: holding the left button of the mouse it is possible to grab and move the map, and by clicking on a station the related information will be shown.

\section{Data analysis}

The preliminary overview of our results reveals that the percentage of high-quality $16 \mathrm{~S}$ fragments assembled (all sequences $>900 \mathrm{bp}$, and chimera-free vs. the total number of contigs assembled) was rather homogeneous across different water depths, ranging from an average of $2.6 \%$ for all MES layers to an average of $4.4 \%$ for all SRF layers (Additional file 1). Interestingly, a high fraction of high-quality fragments $(>6 \%)$ was assembled from both the Antarctic province and the Indian Monsoon Gyres province (from SRF and MES layers, respectively), whereas the lowest fraction of high-quality gene fragments was assembled from the MES layer of the Chile-Peru Current Coastal province. No significant relationship was found

\section{GLOSSARY QUERY SEARCH}
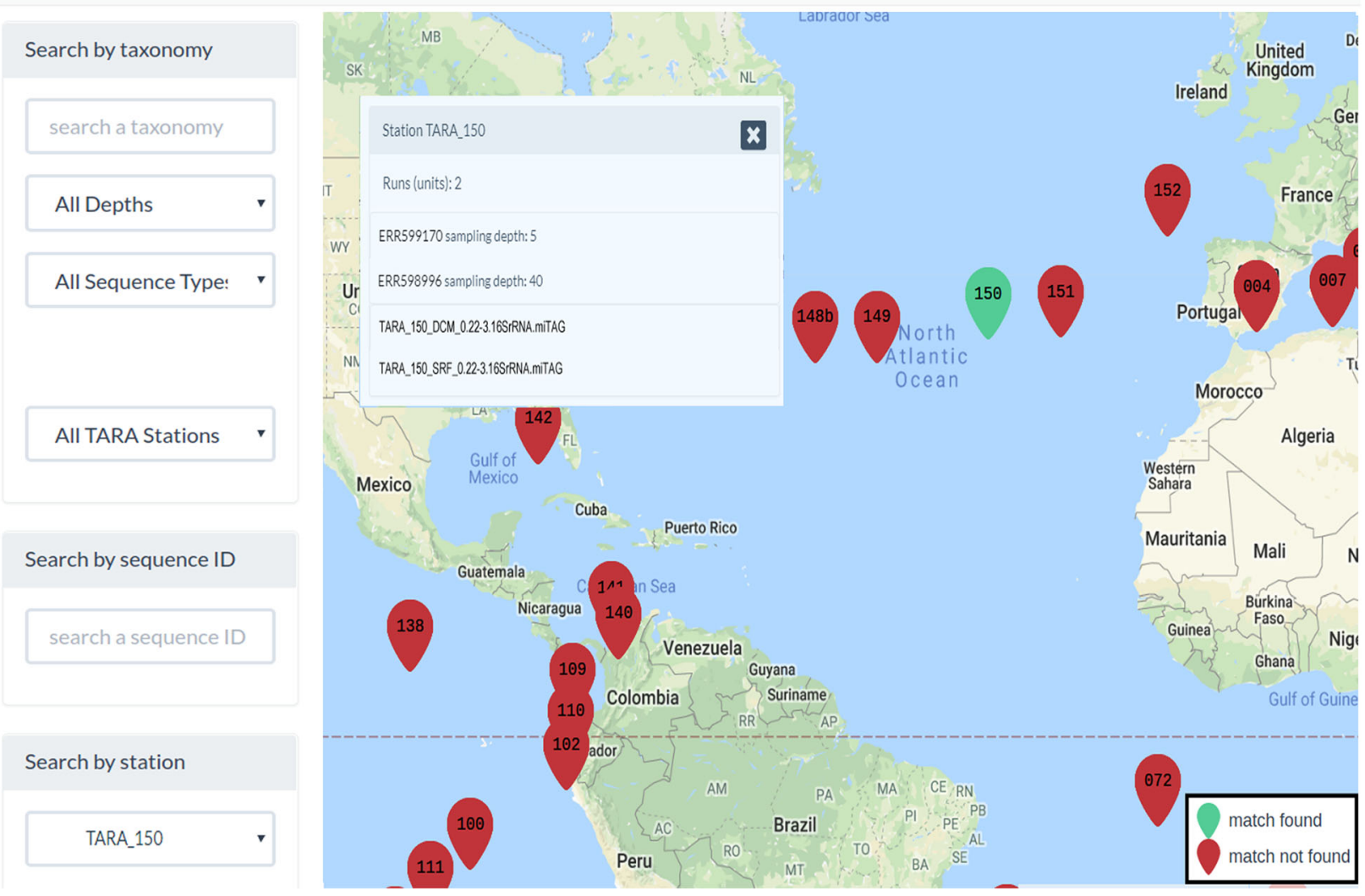

Fig. 5 Example of a search by station. Selecting in the "Search by station" section a Tara station ID (such as "TARA_150", green map pointer), the list of available runs and miTAGs archives (one for each depth) for that station is shown. By clicking on the run name the user will be redirected to the download page of the NCBI SRA section, whereas by clicking on the miTAGs file name it is possible to directly download the FASTA file of the miTAGs sequences for that station and depth 
between the percentage of assembled sequences and the number of high-quality contigs identified.

The number of putatively chimeric contigs represents a variable fraction of the total number of long contigs, ranging from $0 \%$ up to $32 \%$ for contigs longer than $900 \mathrm{bp}$ to more than $60 \%$ of the contigs between 800 and $900 \mathrm{bp}$, across all samples (Fig. 6).

We analysed the geographical distribution of long contigs and represented their relative abundance in terms of number of miTAG per contigs per phylum. Contigs which could not be assigned to any known phylum were also reported as "Unassigned" (Fig. 7). Overall, the average coverage of long contigs reproduced known spatial patterns of prokaryotic abundance in the global ocean where Archaea show a limited distribution in comparison with Bacteria. Interestingly, "Unassigned" contigs are rather frequent and represented in the overall collection, revealing the wideness of still uncovered information on possible prokaryotic phyla.

\section{Discussion}

Previous efforts carried out in the analysis of environmental datasets, such as the Earth Microbiome Project $[16,29]$ and the Global Ocean Sampling [30], produced an astounding amount of sequence data from several environmental samples across the world. Global scale studies can produce an overwhelming amount of data, based on consistent methodological approach, which for the Tara Ocean approach relied also on the use of shotgun genome sequencing strategies. This, in general, allows to collect an important amount of genomic data along with precious informative content [7], thus expanding the targeted sequencing approach previously employed in the Global Ocean Sampling cruises [30]. However, the evaluation of taxonomic diversity across huge datasets with different sampling sizes [19] requires well-calibrated, robust and appropriate methods to get reasonable results. The selection of appropriate sequencing approaches is driven by compromises in terms of

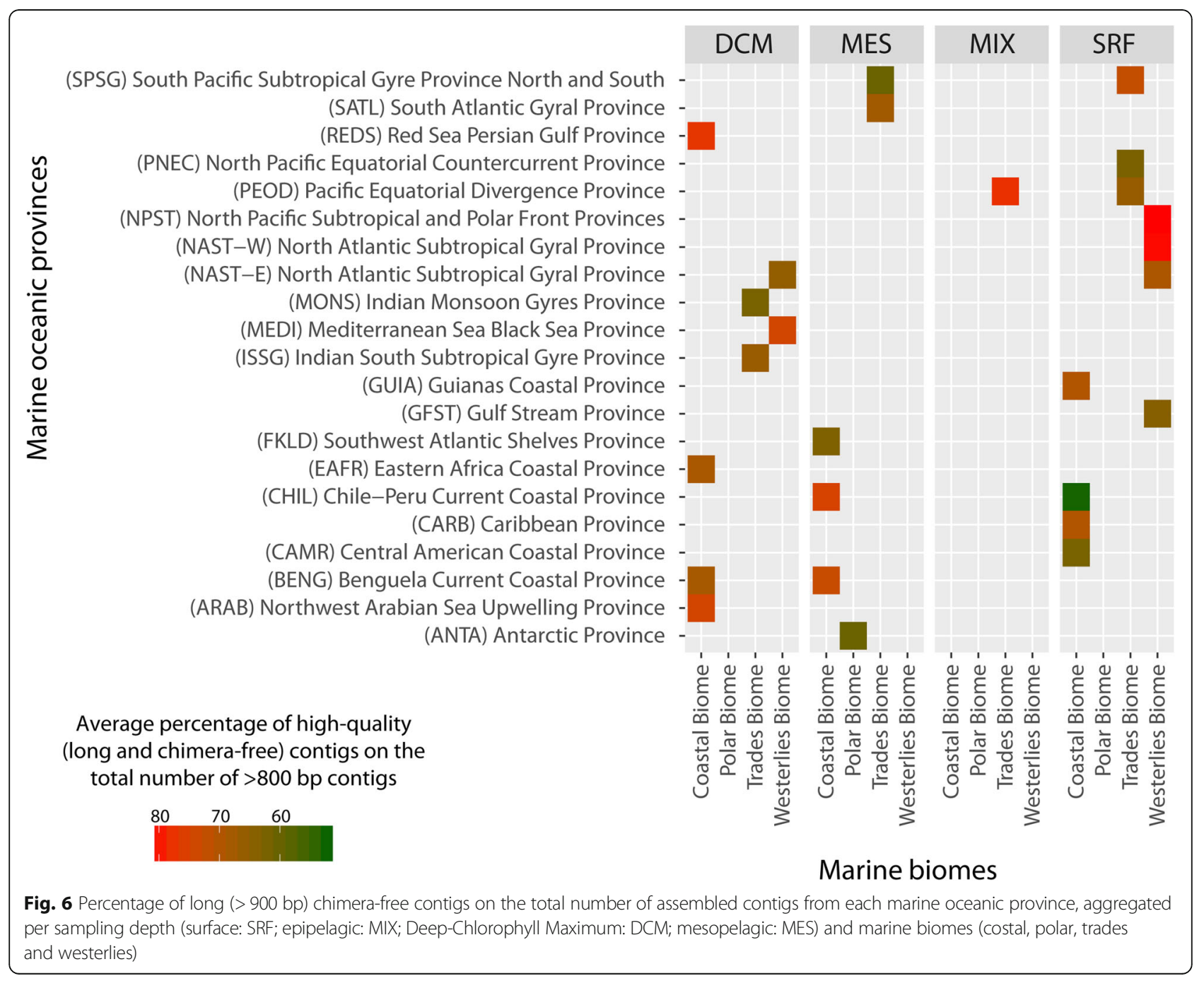




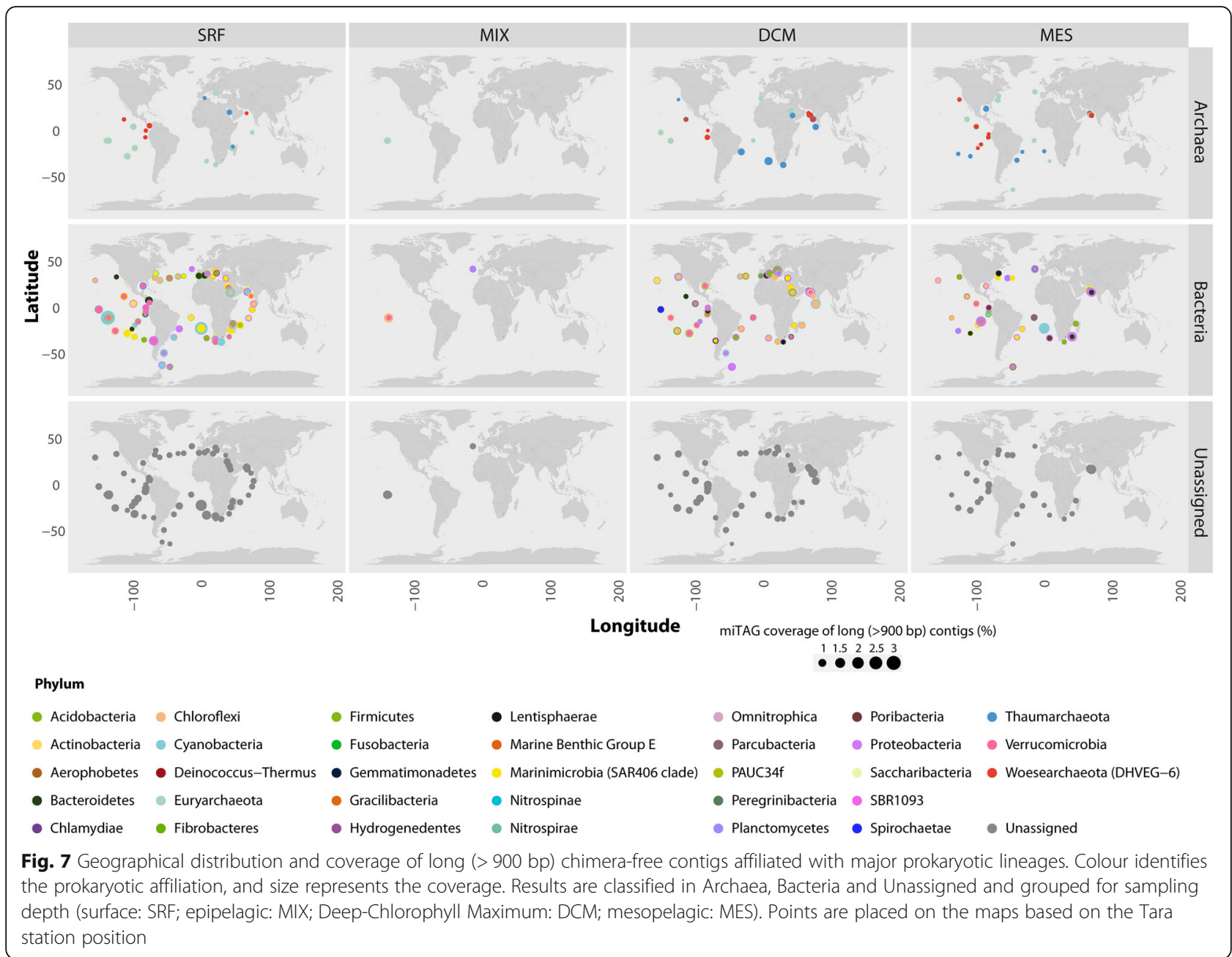

sequencing costs and scientific outputs: the possibility of getting a wider information content with "shotgun" metagenomics than with targeted sequencing may produce novel insights on distribution of species and on their roles in the ecosystems, although the definition of OTUs may be not accurate and capturing the real diversity of prokaryotic taxa may be hard [29, 31].

Rich metadata collections, provided alongside with molecular data associated with environmental 'omic studies, add a further layer of complexity to the analysis. Since the number of similar initiatives is widening (e.g. the Earth Microbiome Project [16, 29], the MicroB3 network [32], the Tara Ocean project [5-7], the Ocean Sampling Day initiative [33], we focused on possible computational approaches to appropriately exploit the information content from shotgun sequencing even in relation with environmental sampling metadata.

To explore the possible computational frameworks that could support appropriate organization and access to these data types, integrating sequence data information and environmental metadata, we designed a dedicated platform, based on a document-oriented NoSQL database management system (DBMS) [27].

We focused on the management and maintenance of information on $16 \mathrm{~S}$ rDNA sequences considering also the possibility to organize collections of heterogeneous quality, since these data type may occur more frequently in shotgun metagenomics and, nevertheless, they represent precious information content to be investigated too.

The starting setup was implemented considering the Tara Ocean dataset, since these data were recently released [6] and they still need a deeper and wider exploration, also exploiting the wide environmental dataset contextually collected. We reconstructed putative $16 \mathrm{~S}$ rDNA contigs by assembling miTAGs, representing $16 \mathrm{~S}$ sequence tags identified and extracted by the Tara Ocean Consortium. The MEGAHIT assembler was chosen to this aim because of its ability to solve $16 \mathrm{~S}$ micro-diversity, thus potentially allowing to discriminate among prokaryotic strains, differing for identities $<97 \%$ in their $16 \mathrm{~S} \mathrm{rDNA}$ sequence [21]. Moreover, the employed strategy allowed maintaining abundance 
information: in fact, the global abundance of major prokaryotic taxa was similar to that highlighted in previous works [33, 34]. Indeed, although the number of long contigs was $<10 \%$ for each library here considered, yet we managed to produce more than 4000 long, chimera-free contigs, which can be used for more complex queries and might allow the exploration of new hypotheses in microbial ecology [7, 35-39].

The presence of putatively chimeric sequences could be due either to miss-assemblies (typically caused by the combined effect of the different degree of variability within the prokaryotic $16 \mathrm{~S}$ locus and the relatively short length of the raw sequences, which can lead to assembly of non-related sequences) or to genuine gene novelty (thus representing $16 \mathrm{~S}$ prokaryotic ribosomal genes with still unclear phylogenetic placement). Although this issue is beyond the scope of the present work, it might be worth of future endeavours to fully characterize the real diversity underlying such datasets. New research direction involving phylogenetic diversity analyses on the present dataset might include a more thorough investigation on the taxonomic assignment of short contigs and raw reads, taking into account the composition of conserved vs. hypervariable regions of prokaryotic $16 \mathrm{~S}$ genes, as well as the presence and characterization of mitochondrial and plastid sequences (which are currently marked as affiliating to either mitochondrial or chloroplast gene sequences according to the SILVA data description).

The BLAST server was implemented using the Sequence Server software [28]. Indeed, this approach allows us to set-up an in-house BLAST service, accessible from the web and with a standard BLAST NCBI-like input and output formats, permitting user driven selections of different data collections. As an example, the possibility to explore in parallel results from the SILVA database [17] and from the in-house implemented collections permits to cross-check sequence relationships as well as to access sequences information along with environmental metadata.

The choice of MongoDB as an alternative approach to more conventional relational DBMS technologies is an emerging trend in bioinformatics, as demonstrated by other similar research projects [40]. This is due to different reasons: i) the huge size of the dataset, demanding replication and data sharing to guarantee safety and performances, as well as scalability; ii) the inhomogeneity of the data collections, which can be much more easily addressed using a schema-less DBMS; iii) the possibility to query geospatial data natively; iv) the possibility to quickly re-organize data collections, allowing for database updates and changes. Since the MongoDB structure and capabilities meet the aforementioned requirements, it has been chosen as the document database for this study.
Similar platforms as the one here proposed are not new to science: complex systems, such as MG-RAST [14], allow researchers to upload, store, analyse and compare metagenomics samples on a global scale. Ribosomal sequence repositories such as the SILVA database [17] also allow scientists to make queries using a proprietary alignment software to identify similar sequences (for a sequence query-based search) or to simply browse the database to download sequences of interest. However, although such web-based systems have become part of standard practices in both shotgun and targeted metagenomics efforts, none of them allows to interactively exploit environmental metadata, which is of paramount importance for ecological studies. Indeed, for instance, MG-RAST allows users to search for specific samples or projects by means of MIMARKS-based metadata [14, 41], which are provided during the submission by data providers, although the environmental metadata are not accessible by straightforward queries on sequence data. The SILVA database [17] provides users with details on sequence data processing and production, but does not store any contextual data in the sequence dataset. The MarRef database [42], on the contrary, provides a rich set of metadata concerning both prokaryotic species features and environmental features and allows BLAST searches, but it only hosts a limited amount of sequence data, concerning few reference species. The most similar, and most recent, implementation of a querable system exploiting the Tara Ocean data is the Ocean Gene Atlas [43, 44], which allows users to compare their own sequence data with either the Tara Ocean Microbiome Reference Gene Catalog (for prokaryotes) or the Marine Atlas of Tara Ocean Unigenes (for eukaryotes). This service allows the navigation and visualization of user-defined sets of nucleotide or amino acid sequences that can be explored based on their functional annotation. GLOSSary, in contrast, allows taxonomic based analyses on $16 \mathrm{~S}$ sequences, supporting investigations on phylogenetic diversity based on this marker. To support the users, the BLAST server embedded in the GLOSSary platform also allows joint analyses versus assembled and unassembled Tara Ocean $16 \mathrm{~S}$ sequences, and those included in the SILVA database, thus supporting comparative analyses of the different outputs in one shot. This is a straightforward approach to detect novel tags from the Tara Oceans collection which are not included in the SILVA collection.

The GLOSSary platform tackles relevant issues in meta "-omics" from environmental data starting from the organization of heterogeneous $16 \mathrm{~S}$ rDNA data and from their associated metadata, favouring efficient queries on large amount of information and their analysis by suitable graphical approaches. Instead of replicating already-existing frameworks, GLOSSary also allow for 
BLAST-like sequence search and comparison, which also integrates a well-established reference database, as well as metadata-informed query of prokaryotic taxa on a global scale.

Although this initial effort is now presented as a framework which embeds the Tara Oceans data, its underlying objective is to expand with additional dataset from similar resources, aiming at a comprehensive collection that could support the exploration of prokaryotic taxonomic diversity integrated with their environmental characterization.

\section{Conclusions}

The current release of the GLOSSary platform aims at favouring the exploration of 16S rRNA gene sequences from large-scale environmental samplings. The database is designed for friendly scalability and multifaceted data uploading. The more conventional sequence based investigations are supported by views that favour environmental metadata exploitation.

The current release of the platform includes $16 \mathrm{~S}$ data processed from the miTAG collection released by the Tara Ocean expedition and allows not only to identify the location of specific prokaryotic families, but also to investigate their coexistence with user-driven queries, allowing explorations of ecological and phylogenetic issues.

Future efforts will be focused on the inclusion of more $16 \mathrm{~S}$ data from similar ongoing efforts, to establish a reference resource for the investigation of prokaryotic diversity embedded in the environmental context.

\section{Availability and requirements}

Project name: GLOSSary - the GLobal Ocean 16S

Subunit web accessible resource.

Project home page: http://bioinfo.szn.it/glossary/

Operating system(s): Platform independent (web-server).

Programming language: Python (database query system), HTML5 and Javascript (frontend).

Other requirements: none (web-server).

License: none applicable.

Any restrictions to use by non-academics: no restrictions.

\section{Additional files}

Additional file 1: Table summarizing the resulting contigs obtained from the Tara miTAGs assembly. (DOCX $33 \mathrm{~kb}$ )

Additional file 2: Visualization of the unevenness of the Tara Ocean sampling effort. (JPG $147 \mathrm{~kb}$ )

\section{Abbreviations}

DBMS: Database management system; GLOSSary: The GLobal Ocean $16 \mathrm{~S}$ Subunit web accessible resource

\section{Acknowledgements}

We thank Chris Bowler (Institut de Biologie de l'Ecole Normale Supérieure (IBENS), France) and Daniele ludicone (Stazione Zoologica "Anton Dohrn", Naples) for updates on the Tara effort and on the status of available collections. We thank Pier Luigi Buttigieg (MPI for Marine Microbiology) and the task force on "Omics observatories" for useful discussions on environmental metagenomics.

\section{Funding}

The present work was supported by the SZN (Stazione Zoologica "Anton Dohrn") flagship project BIOINforMA: BIOINformatics for MArine Biology, which also covered publication costs for this manuscript.

Availability of data and materials

The GLOSSary database and service is publicly available at http://bioinfo.szn.t/ glossary/.

\section{About this supplement}

This article has been published as part of BMC Bioinformatics Volume 19 Supplement 15, 2018: Proceedings of the 12th International BBCC conference. The full contents of the supplement are available online at https:// bmcbioinformatics.biomedcentral.com/articles/supplements/volume-19supplement-15.

\section{Authors' contributions}

MM designed the MONGOdb database and the associated query system. ChC and MS organized the BLAST partition of the platform, MT processed the sequence data, MT and ChC checked and validated raw miTAG sequences; $\mathrm{CiC}, \mathrm{AD}$ and $\mathrm{RD}$ supervised the entire scientific effort and tested the platform. $M L C$ conceived the strategy and the platform and supervised the entire effort, MM realized the schema-less database and made the computational effort concrete, MT proposed and designed the analysis on $16 \mathrm{~S}$ data. All authors read and approved the final manuscript.

Ethics approval and consent to participate

Not applicable.

Consent for publication

Not applicable.

\section{Competing interests}

The authors declare that they have no competing interests.

\section{Publisher's Note}

Springer Nature remains neutral with regard to jurisdictional claims in published maps and institutional affiliations.

\section{Author details}

'Stazione Zoologica "Anton Dohrn", Villa Comunale, 80121 Naples, Italy. 2Dipartimento di Scienze della Vita e dell'Ambiente, Polytechnic University of Marche, Via Brecce Bianche, 60131 Ancona, Italy. ${ }^{3}$ Dipartimento di Scienze e Ingegneria della Materia, dell'Ambiente ed Urbanistica, Polytechnic University of Marche, Via Brecce Bianche, 60131 Ancona, Italy. ${ }^{4}$ Dipartimento di Agraria, University of Naples "Federico II", via Università 100, 80055 Portici, Italy.

Published: 30 November 2018

References

1. Church MJ, et al. Physical forcing of nitrogen fixation and diazotroph community structure in the North Pacific subtropical gyre. Glob Biogeochem Cycles. 2009;23(2). https://doi.org/10.1029/2008GB003418.

2. Nedwell D, Rutter M. Influence of temperature on growth rate and competition between two psychrotolerant Antarctic bacteria: low temperature diminishes affinity for substrate uptake. Appl Environ Microbiol. 1994:60(6):1984-92.

3. Fuhrman JA, Cram JA, Needham DM. Marine microbial community dynamics and their ecological interpretation. Nat Rev Microbiol. 2015; 13(3):133.

4. Chafee $\mathrm{M}$, et al. Recurrent patterns of microdiversity in a temperate coastal marine environment. ISME J. 2017;12(1):237. 
5. Karsenti E, et al. A holistic approach to marine eco-systems biology. PLoS Biol. 2011;9(10):e1001177.

6. Pesant $\mathrm{S}$, et al. Open science resources for the discovery and analysis of Tara oceans data. Scien Data. 2015;2:150023.

7. Sunagawa $S$, et al. Structure and function of the global ocean microbiome Science. 2015;348(6237):1261359.

8. Tully BJ, et al. 290 metagenome-assembled genomes from the Mediterranean Sea: a resource for marine microbiology. Peer J. 2017;5: e3558.

9. Tully BJ, Graham ED, Heidelberg JF. The reconstruction of 2,631 draft metagenome-assembled genomes from the global oceans. Sci Data. 2018;5: 170203.

10. Roux S, et al. Ecogenomics and potential biogeochemical impacts of globally abundant ocean viruses. Nature. 2016;537(7622):689.

11. Delmont TO, et al. Nitrogen-fixing populations of Planctomycetes and Proteobacteria are abundant in surface ocean metagenomes. Nat Microbiol. 2018:3:804-13.

12. Sun $\mathrm{S}$, et al. Community cyberinfrastructure for advanced microbial ecology research and analysis: the CAMERA resource. Nucleic Acids Res. 2010; 39(suppl_1):D546-51.

13. Huse SM, et al. VAMPS: a website for visualization and analysis of microbial population structures. BMC Bioinf. 2014;15(1):41.

14. Meyer $\mathrm{F}$, et al. The metagenomics RAST server-a public resource for the automatic phylogenetic and functional analysis of metagenomes. BMC Bioinf. 2008;9(1):386.

15. QIITA server. http://qiita.microbio.me

16. Gilbert JA, Jansson JK, Knight R. The earth microbiome project: successes and aspirations. BMC Biol. 2014;12(1):69.

17. Quast C, et al. The SILVA ribosomal RNA gene database project: improved data processing and web-based tools. Nucleic Acids Res. 2012;41(D1):D590-6.

18. Tara Ocean portal at the European Nucleotide Archive https://www.ebi.ac. uk/ena/data/view/PRJEB7988. Accessed 15 Nov 2017.

19. Logares $\mathrm{R}$, et al. Metagenomic $16 \mathrm{~S}$ rDNA Illumina tags are a powerful alternative to amplicon sequencing to explore diversity and structure of microbial communities. Environ Microbiol. 2014;16(9):2659-71.

20. Li D, et al. MEGAHIT: an ultra-fast single-node solution for large and complex metagenomics assembly via succinct de Bruijn graph. Bioinformatics. 2015;31(10):1674-6.

21. Vollmers J, Wiegand S, Kaster A-K. Comparing and evaluating metagenome assembly tools from a microbiologist's perspective-not only size matters! PLoS One. 2017;12(1):e0169662.

22. The R Team. R: A language and environment for statistical computing. 2013.

23. Parks DH, et al. CheckM: assessing the quality of microbial genomes recovered from isolates, single cells, and metagenomes. Genome Res. 2015; 25(7):1043-55.

24. Schmieder R, Edwards R. Quality control and preprocessing of metagenomic datasets. Bioinformatics. 2011;27(6):863-4.

25. Caporaso JG, et al. QIIME allows analysis of high-throughput community sequencing data. Nat Methods. 2010;7(5):335.

26. Rognes T, et al. VSEARCH: a versatile open source tool for metagenomics. PeerJ. 2016;4:e2584.

27. Chodorow K, Dirolf M, Mongo DB. The Definitive Guide. Sebastopol: O'Reilly Media, Inc.; p. 216

28. Priyam A, et al. Sequenceserver: a modern graphical user interface for custom BLAST databases. Biorxiv. 2015:033142.

29. Thompson $L R$, et al. A communal catalogue reveals Earth's multiscale microbial diversity. Nature. 2017;551(7681). https:/doi.org/10.1029/2008GB003418.

30. Rusch DB, et al. The sorcerer II global ocean sampling expedition: northwest Atlanticthrough eastern tropical Pacific. PLoS Biol. 2007;5(3):e77.

31. Amir A, et al. Deblur rapidly resolves single-nucleotide community sequence patterns. MSystems. 2017;2(2):e00191-16.

32. MicroB3 network. https://www.microb3.eu/ Accessed 01 Mar 2018.

33. Kopf A, et al. The ocean sampling day consortium. Gigascience. 2015;4(1):27.

34. Zinger $L$, et al. Global patterns of bacterial beta-diversity in seafloor and seawater ecosystems. PLoS One. 2011;6(9):e24570.

35. Hugoni M, et al. Structure of the rare archaeal biosphere and seasonal dynamics of active ecotypes in surface coastal waters. Proc Natl Acad Sci. 2013;110(15):6004-9.

36. Danovaro $R$, et al. A submarine volcanic eruption leads to a novel microbial habitat. Nat Ecol Evol. 2017;1(6):0144.
37. De Wit R, Bouvier T. 'Everything is everywhere, but, the environment selects'; what did baas Becking and Beijerinck really say? Environ Microbiol. 2006;8(4):755-8

38. Haggerty JM, Dinsdale EA. Distinct biogeographical patterns of marine bacterial taxonomy and functional genes. Glob Ecol Biogeogr. 2017;26(2): 177-90.

39. Gonnella $\mathrm{G}$, et al. Endemic hydrothermal vent species identified in the open ocean seed bank. Nat Microbiol. 2016;1(8):16086.

40. Stajdohar $M$, et al. dictyExpress: a web-based platform for sequence data management and analytics in Dictyostelium and beyond. BMC Bioinf. 2017; 18(1):291.

41. Yilmaz $P$, et al. Minimum information about a marker gene sequence (MIMARKS) and minimum information about any ( $\mathrm{x}$ ) sequence (MIXS) specifications. Nat Biotechnol. 2011;29(5):415.

42. MarRef database. https://mmp.sfb.uit.no/databases/marref/. Accessed 1 Mar 2018.

43. Tara Ocean Gene Atlas: http://tara-oceans.mio.osupytheas.fr/ocean-gene-atlas/. Accessed 1 Mar 2018.

44. Villar $E_{\text {, et }}$ al. The Ocean Gene Atlas: exploring the biogeography of plankton genes online. bioarXiv. 2018. https://doi.org/10.1101/271882.

\section{Ready to submit your research? Choose BMC and benefit from:}

- fast, convenient online submission

- thorough peer review by experienced researchers in your field

- rapid publication on acceptance

- support for research data, including large and complex data types

- gold Open Access which fosters wider collaboration and increased citations

- maximum visibility for your research: over $100 \mathrm{M}$ website views per year

At BMC, research is always in progress.

Learn more biomedcentral.com/submissions 